\title{
L'exposé de la Doctrine de la Science de 1805
}

\author{
MANUEL JIMÉNEZ-REDONDO
}

Université de Valencia

L'exposé de la Doctrine de la science de 1805 de Fichte est un texte qui, s'il exerce sur le lecteur d'aujourd'hui une rare fascination, n'en est pas moins, dans son ensemble, presque inintelligible. C'est dû au fait, entre autres, que Fichte entre dans cette exposition sans plan déterminé, le plan n'émergeant qu'au long de l'exposé même, non sans procéder qui plus est à un grand nombre de retours. S'ajoute à cela qu'au moins à partir du début de la seconde moitié de l'exposé, la ligne d'argumentation se montre diffuse et entrecoupée et devient, en outre, de plus en plus aporétique. Dans ce qui suit, je voudrais proposer une reconstruction de la ligne argumentative de l'exposé, où la philosophie de la conscience opère tant de dissections du concept de la conscience et du savoir que, dans ce qui en émerge, la pensée ancienne et moderne ainsi que plusieurs motifs de la pensée contemporaine se donnent la main.

On va parler du savoir - naturellement, cela ne peut se faire, Fichte le dit dans la première conférence, qu'en sachant, c'est-à-dire de l'intérieur du savoir lui-même. Par conséquent, notre dire et cela même que nous disons doivent se montrer comme étant la même chose, comme s'épuisant identiquement l'un dans l'autre.

Parler du savoir, c'est parler de l'événement d'une différence. C'est la différence entre l'être (Seyn) et l'existence (Existenz), entre l'être et le se-montrer de l'être, ou, puisque le savoir s'exprime ou peut s'exprimer dans un énoncé, entre le sujet de l'énoncé et le est de l'énoncé, dans la triple signification possible de ce est: au sens de la prédication (l'arbre est vert), de l'identité (l'arbre dont je parle maintenant est le même dont je parlais hier) ou encore au sens de l'existence (l'arbre est, l'arbre existe).

Nous cherchons à savoir ce qu'est le savoir en soi. La réponse devra avoir la forme suivante: en soi, le savoir est ceci ou cela. Par conséquent, savoir ce qu'est le savoir en soi devra consister avant tout à savoir ce qu'est ce est avec lequel le savoir s'identifie. Car, chaque fois que nous avons un est, s'il est le 
est d'un énoncé vrai, nous avons un véritable savoir : le véritable savoir et ce est s'épuisent identiquement l'un dans l'autre. Répondre à la question de savoir ce qu'est le savoir en soi, c'est répondre à la question: " qu'est-ce que ce est? "Une fois que l'on a répondu à cette question, on peut considérer comme accomplie la tâche de la doctrine de la science (Frage beantwortet und die WL geschlossen) ${ }^{1}$.

Ce est - dit Fichte - n'est pas simplement l'objet mis dans la sphère de l'être, mais il est quelque chose de plus; il est cet objet se montrant, son êtredehors, son ex-sistence, il est äussere Umfassung und Umgebung, entourage et environnement externes ${ }^{2}$. Quand nous pensons à l'être - dit Fichte -, nous le pensons comme étant de soi, en soi et par soi; il serait le même s'il n'y avait aucun savoir de lui, et avec cela, nous avons épuisé l'idée d'être ${ }^{3}$; l'être est refermé sur lui-même, il s'épuise en soi-même et on ne voit absolument pas comment il pourrait y avoir un dehors à partir duquel on pourrait dire qu'il est. Car ce dehors de l'être contredit le concept d'être: il serait le rien.

Cependant, en disant qu'il n'y a pas un dehors de l'être, en disant que l'être est quelque chose de fermé et qui s'épuise en lui-même, nous nous sommes déjà placés et nous nous mouvons déjà en son dehors; voilà le est, un est secret qui paraît vouloir se cacher, disparaître, et qui, de cette manière, se montre comme étant tout-à-fait différent de l'être, comme rien ${ }^{4}$. C'est pourquoi, justement quand nous parlons de cette façon, il se cache à nous une fois encore parce qu'en disant que le est est ceci ou cela, nous mettons le est dans la sphère de l'être, et de cette façon nous avons oublié le est. Le est s'échappe, s'efface lui-même. Dans la philosophie transcendantale, cette confusion ou cet oubli - dit Fichte - est l'erreur fondamentale, source de toutes les erreurs. Arriver à savoir ce qu'est le savoir en soi ${ }^{5}$ devra consister à expliquer ce hiatus entre l'être et l'existence; nous voulons " arriver à comprendre avec clarté la loi par laquelle cette scission se produit et comment elle se produit ${ }^{6}$ ".

Comme nous l'avons vu précisément au moment où le est se cachait à nous, le est n'est qu'en construisant son propre être qualitatif interne en

1. Johann Gottlieb FiCHTE, Gesamtausgabe, II, 9, 1993, p. 185.

2. Ibid., p. 183.

3. Ibid., p. 186.

4. Pour me limiter à deux exemples, Fichte traite dans ce cours, mais sur des registres bien plus diversifiés, le même sujet que celui développé par M. Heidegger dans sa fameuse conférence de 1929, "Qu'est-ce que la métaphysique?" et par M. Merleau-Ponty dans Le visible et l'invisible (voir en particulier "Interrogation et dialectique").

5. FICHTE, op. cit., p. 187.

6. Ibid., p. 203. 
opposition à la fois à $l$ 'être et à son propre être. Existence veut dire, d'abord, que l'être reste dehors, reste exposé à la vue; l'existence de l'être, étant ce qu'elle est seulement en opposition à l'être lui-même, est l'être comme tel, l'être en tant qu'être, comme se montrant par opposition à ce qui n'est pas l'être (et aussi en opposition à son propre être) (quatrième conférence).

Cela veut dire en outre que si l'existence n'est qu'en opposition à l'être et, en ce sens, n'est pas seulement l'être comme tel, elle ne peut à son tour l'être qu'en opposition à son propre être qui apparaît de cette façon à l'intérieur d'elle-même et en opposition à elle-même; l'existence n'est que comme existence, ou en tant qu'existence, c'est-à-dire l'existence est ce comme ou ce en-tant-que $(A l s)$. L'existence est ce $A l s$ qui apparaît à lui-même, c'est-àdire le $A l s$ n'est que comme $A l s$; le $A l s$ a toujours la structure d'un $A l s$ als $A l s$ (troisième conférence).

On peut dire la même chose d'une autre manière encore. Le est est seulement le est de quelque chose qui est, si, quand nous le prononçons (c'està-dire quand la différence signalée par le terme est se produit), la chose reste pour la vue en tant que telle, en même temps que ce rester pour la vue reste à son tour pour la vue, c'est-à-dire si ce est est à son tour. De cette façon, on peut aussi dire que ce est qui n'est rien d'autre que l'étant comme tel, est redoublement, image, Bild. Mais il s'agit d'une image qui, à son tour, n'est qu'en construisant son propre être comme image, par opposition à l'être dont elle est l'image; l'image n'est pas sans se faire image de soi-même.

Et, de façon dérivée, on peut dire aussi que l'existence, le est, n'est qu'une conscience qui n'est pas sans conscience de soi (A/A) et qui n'est conscience que par opposition à un objet $(B)$ en général. Nous avons, par conséquent, la structure A/A x B qui est la structure de la conscience d'un objet, conscience qui n'est donc pas possible sans conscience de soi. Cependant, cela ne veut pas dire que les concepts ontologiques et les concepts qui se rapportent à la structure de l'énoncé puissent être réduits à des concepts qui se rapportent à la relation sujet-objet, mais, au contraire, que les concepts qui se rapportent à la relation sujet-objet, à la conscience, à la conscience de soi et au moi, peuvent être réduits aux concepts ontologiques, moyennant des concepts qui se rapportent à l'énoncé.

Le savoir est donc en soi ce est, absolute sumptum; le savoir est l'existence absolue. Or, la thèse fondamentale (et surprenante) que Fichte se propose de développer dans cet exposé, c'est que l'on ne peut pas voir ce que peut être cette existence absolue, si elle n'est pas l'existence de l'absolu (deuxième conférence), l'existence de Dieu. 


\section{Première tentative de démonstration: l'absolu apparent}

Jusqu'ici, j'ai fait référence aux motifs fondamentaux des quatre premières conférences. Les considérations sur l'être, contenues dans les conférences 5 et 6 , sont des passages dans lesquels toute tentative de vouloir reconstruire la ligne argumentative de l'exposé de Fichte pourrait facilement se fourvoyer. Dans la sixième conférence, Fichte présente ses excuses pour l'obscurité de l'exposition. Mais dans la septième conférence, il prend ce qu'il appelle un raccourci (Wegverkürzung) qui nous conduit " directement et sans diminution de clarté au théorème suprême ", " au point proprement dit et suprême ", à la démonstration de la thèse fondamentale. Dans les conférences 8 à 10, Fichte complète cette démonstration et analyse ses résultats.

Ce " théorème suprême 》 affirme ceci : «que l'existence en elle-même ne suive pas est la condition absolue de sa propre existence; et si l'existence suivait en elle-même (c'est-à-dire si elle pouvait être intelligée comme une conséquence), alors elle serait par là complètement supprimée et anéantie ?."

Fichte livre en deux temps la " démonstration " de ce théorème suprême qui établit que si le savoir, en soi-même, est l'existence absolue, alors cette existence absolue n'est autre que l'existence de l'absolu.

L'existence n'est qu'en tant qu'existence, c'est-à-dire, l'existence, en s'opposant à l'être, n'est qu'en construisant son être propre en même temps qu'elle s'oppose à lui. Le premier moment de la démonstration de Fichte est négatif et consiste à dire que si, en elle-même, l'existence devait trouver une raison d'elle-même, cette raison serait sans doute une raison en tant que telle, ce qui présuppose précisément déjà l'existence parce que l'existence est ce en tant que. Et si cette existence présupposée devait trouver en ellemême, et à son tour, une raison, celle-ci devrait être une raison en tant que telle, ce qui présupposerait précisément déjà l'existence, et ainsi in infinitum. L'existence est donc Nicht-Folge, Abgrund.

Le deuxième moment est positif. L'existence est une relation, mais une relation qui ne sort pas d'elle-même, qui n'est pas une médiation. Elle présente un caractère immédiat, qui est une identité interne de deux relata. Et ces deux relata ne peuvent être intérieurement un que par le biais d'un en tant que $(A l s)$. Ici, la relation ne l'est que comme un tiers entre les deux relata, parce que les deux relata sont déjà cette relation même, c'est-à-dire parce que la relation est déjà le premier et le deuxième relatum, donc parce que ses relata sont déjà la relation, et parce que celle-ci est déjà les relata et le fondement de leur relation. La relation ne découle de rien qui lui préexisterait.

7. Ibid., p. 209. 
Pour rendre compte de cette relation, dit Fichte, on se sert dans le langage courant de la notion de lumière (intérieure). Le savoir est le est de l'objet qui est, à son tour, comme le est de ce est, sans toutefois sortir de lui, ou, en étant ces deux, il est l'un et le même dehors. Dans le cas où l'objet est le savoir, le savoir, en se sachant lui-même, est le est de ce est, tout en étant en même temps le est de ce est. Et une fois que nous avons ce concept dans son unité organique, nous pouvons dire: ce n'est pas la relation absolue qui est la lumière, mais c'est la lumière qui est la relation absolue, ce n'est pas le $A l s$, la relation, qui suscite la lumière, mais c'est la lumière qui suscite le en tant que dans lequel quelque chose (qui dans notre cas est le savoir luimême, ou maintenant la lumière elle-même) est en tant que telle et reste en tant que telle la lumière. La lumière est en elle-même absolue, elle n'a besoin d'aucun porteur qui la précèderait parce qu'elle est ce qui porte tout porteur en tant que tel. Et rien ou personne qui ne soit porteur en tant que tel de la lumière, ne peut la porter. Par conséquent, tout porteur suppose déjà à son tour la lumière en tant que porteur. La lumière a dû être produite à partir du néant parce qu'elle n'a pas été produite, et parce que c'est à partir d'elle qu'a été faite toute chose qui peut être considérée comme telle, y compris elle-même, et hors d'elle rien n'a été fait. Et si on prend en considération un néant préalable, alors à ce néant, à ce non-quelque-chose en tant que tel devrait précéder la lumière, c'est-à-dire qu'il ne pourrait être qu'à l'intérieur de la lumière. La lumière, une fois qu'elle est ne peut pas ne pas être, et il n'y a pas un dehors d'elle, parce que c'est elle qui suscite l'être dont elle est le en tant que tel auquel elle est nécessairement rattachée. Et cependant la lumière, dans son caractère absolu, dans son caractère ultime et dans son agilité pure, est aussi facticité pure, grundlose Wirklichkeit.

Or, cet être de la lumière est l'" énigmaticité " pure ou l'incompréhensibilité pure, la non lumière. En apercevant (ersehen) son propre en-soi, un en-soi qui ne peut être que le résultat du fait qu'elle se voie (sehen), la lumière dans la lumière cesse de l'être, elle n'est déjà plus lumière. En se trouvant devant elle-même, elle s'anéantit, cesse d'être lumière et ne voit plus. «L'auto-anéantissement de la lumière découle de ce qu'elle est la genèse de son être absolu en elle-même ${ }^{8}$. " Si la lumière est en elle-même absolue, et consiste en une agilité pure, en un acte pur sans agent, en une Tathandlung, en l'acte pur d'engendrer son propre être, c'est en ce même caractère absolu que consiste l'être dont la pensée est l'anéantissement de la lumière. C'est "précisément dans l'acte de s'engendrer elle-même ${ }^{9}$ " que la lumière s'anéantit dans cette génération, en faisant de cet acte l'acte de son 
propre être, ce qui veut dire: l'acte d'un étranger, d'une énigme (de quelque chose qui est essentiellement non-lumière), puisqu'elle ne peut pas considérer que cet acte de génération absolue procède d'elle. De qui alors? De l'absolu. L'absolu existe donc comme lumière au point où la lumière, en se comprenant comme conséquence absolue, cesse de l'être. L'existence absolue est donc l'existence de l'absolu.

La lumière qui jaillit du néant de soi (le je pense qui soutient le tout, mais que rien ne soutient), qui, une fois qu'elle est, ne peut pas ne pas être, mais qui en même temps ne peut pas suffire à cette condition et qui, en elle-même, ne peut être considérée que comme conséquence absolue, "s'engendre ellemême simplement pour devenir cette existence [à savoir celle de l'absolu]; sa génération, en son dedans, n'est en soi absolument rien, mais elle est seulement la manière externe et la condition externe sine qua non de l'existence de l'absolu. Mais alors, il est complètement faux d'affirmer que c'est la lumière même qui s'engendre: ainsi le paraît-il seulement lorsqu'elle reste en soi-même; mais quand, en s'anéantissant elle-même, elle sort de soi, c'est l'absolu même qui, par un acte libre, inscrutable, engendre sa propre existence [...]. Et ainsi, ce n'est pas Dieu qui existe parce que la lumière est, mais c'est la lumière qui existe parce que Dieu existe et son existence est nécessairement la lumière [... ${ }^{10}$. » Mais l'existence de Dieu découle nécessairement de son essence.

\section{Deuxième tentative de démonstration: l'absolu de la foi}

Rien ne permet d'interpréter le contenu des conférences sept à dix dans un sens purement "éristique ", comme si Fichte l'eût seulement exposé pour pouvoir le réfuter. Ces conférences ne sont pas seulement le meilleur de tout l'exposé de Fichte, de surcroît c'est en elles qu'il s'exprime avec le plus d'enthousiasme.

Cependant, en cours d'exposition, Fichte se rend compte que sa démonstration du théorème fondamental n'est qu'un sophisme. À partir de la onzième conférence, une fois que l'échec de la première tentative de démonstration devient évident, l'exposé se divise en quatre autres tentatives, à chaque fois différentes, de démontrer le théorème fondamental. À partir de cette onzième conférence, l'exposé de Fichte est difficilement intelligible si on veut le comprendre dans un sens linéaire. Néanmoins, l'exposé est sans heurt, en ce sens du moins où le professeur n'éprouve ni hâte, ni la nécessité de recourir à un plan prédéterminé. 
L'objection à laquelle Fichte a dû répondre est la suivante: si nous appelons réflexion absolue cette auto-projection de la lumière sur l'idée de l'absolu, cet absolu est le résultat de cette réflexivité absolue, c'est-à-dire qu'il n'est pas autre chose que l'objectivation dans la lumière, et par la lumière, de l'auto-génération de la lumière. Il est stehendes Licht, lumière arrêtée, lumière qui s'arrête elle-même, chose inerte, nullement absolue, mais purement relative. Toutefois, ce n'est pas la lumière qui fait l'absolu, mais l'absolu qui fait la lumière. C'est l'absolu qui est la raison de la facticité de la lumière, raison devant laquelle la lumière s'anéantit.

Si nous nous en tenons énergiquement au point où nous sommes, et où nous prenons une identité maintenant, qui n'est rien d'autre que le fait de ne pas laisser valoir l'apparence contre la vérité, nous savons alors que cette lumière factuelle est un absolu et un pur réaliser de sa propre essence de rien et à partir de rien, même contre son stehendes Seyn ou contre toute objectivation qui offre, ou peut offrir, l'apparence d'être l'absolu. Cette lumière devenue maintenant pour elle-même un factum absolu et dernier, mais qui ne laisse pas valoir son stehendes Seyn comme l'absolu, est la foi. Et c'est à la foi que l'absolu se donne véritablement dans le caractère qu'il a d'être essentiellement non-lumière pour la lumière. Le savoir, l'existence absolue est donc l'existence de l'absolu de cette foi qui ne laisse pas valoir l'apparence d'absolu, parce que l'absolu ne peut pas être le résultat de ne pas vouloir regarder la facticité dernière de la lumière (de ne pas vouloir regarder la lumière dans sa facticité dernière) ou ne peut pas être confusion de la lumière avec sa propre facticité. L'absolu devant lequel la lumière s'anéantit véritablement, l'étranger absolu face auquel la lumière cesse véritablement d'être lumière, se donne à la foi; il se donne seulement au regard qui regarde cette facticité dernière comme ce qu'elle est, en son apparence d'être l'absolu, et qui ne laisse pas valoir cette apparence contre la vérité. C'est donc la foi qui divise l'absolu comme auteur de la lumière, mais non pas de cette lumière qui découlerait de ce qui se montrait à la fin comme n'étant qu'un produit de la réflexivité absolue, mais de la lumière dans laquelle l'absolu lui-même baigne, au-delà de toute facticité. Le moi pur et inobjectivable est cette lumière de la foi, revenant à elle-même. Le moi de la foi est le représentant immédiat de l'absolu.

Mais au long des conférences 11 à 13 , il devient de plus en plus clair que la foi en laquelle nous nous sommes maintenant convertis, est évidemment quelque chose qui peut être ou qui peut aussi ne pas être, c'est-à-dire qu'elle est quelque chose qui, en elle-même, intérieurement, se trouve dans l'impossibilité de voir une quelconque nécessité de soi ou de l'absolu qui se donne à elle, et qui en ce sens est libre. De la non-foi en l'absolu apparent à 
la foi en l'absolu qui se donne lui-même à la foì, il y a un saut qui fait de cet absolu quelque chose de dépendant de ce que l'on veut faire ou non ce saut.

Fichte abandonne cette foi dans la conférence 14. Mais il s'agit d'un abandon qui se produit de l'intérieur de la foi même. Si l'existence absolue, dont le moi dépend, doit se comprendre comme existence de l'absolu, alors seul l'absolu peut exister; il occupe, pour ainsi dire, toute l'existence. Et il existe nécessairement en tant qu'absolu (puisque l'existence n'est qu'en tant $q u$ 'existence, ou l'existence n'est que l'être en tant que tel). Par conséquent, la forme même qu'est l'existence est de soi nécessaire. Or, nous avons vu que la foi n'est rien de cela à ses yeux. Le Dieu de la foi radicalisée et intellectualisée de Luther dissout des notions de l'absolu qui ne sont pas autre chose que des confusions de la lumière avec elle-même. Mais au fond, celle-ci n'est autre que facticité. Elle n'est rien d'autre qu'une manière distincte pour la lumière de se confondre avec soi. C'est l'opinion de Fichte. Aussi le double absolu de la foi (celui que la foi postule moyennant la non-foi en l'apparence, et celui qui se donne à la foi) est-il généré par la lumière, précisément par la lumière en laquelle nous consistons ici, qui a déjà cessé de considérer son propre produit comme valide. Si l'existence doit pouvoir se comprendre comme existence de l'absolu, "la facticité, c'est-à-dire toute la réalité, envisagée hors de son fondement, doit dans la racine de son unité pouvoir être déduite de la nécessité, ou de l'essence ${ }^{11}{ }$. Nous abandonnons donc proprement la foi.

\section{Troisième tentative: existence et concept, Dieu et le monde}

Dans la première tentative de démonstration du théorème fondamental, nous disions que si nous cherchions pour le $A l s$ une raison qui le précèderait, alors celle-ci devrait être une raison en tant que telle; par conséquent, le $A l s$ précède toute raison. C'est le $A l s$ qui, dans tous les cas, porte le fondement avec soi, et si l'existence absolue est l'existence de Dieu, alors l'existence de Dieu est d'être fondement, fondement de la lumière hors de la lumière, ou alors elle n'est plus fondement. Ajoutons maintenant: il y a donc un Dass (quod) de ce $A l s$, et ce Dass est ce qu'il y a de premier. Il précède le $A l s$ de sorte que, si le $A l s$ est la lumière, ce Dass est avant toute lumière. Il précède même le néant en tant que tel. Une fois de plus il s'agit donc de la facticité absolue de la lumière. La lumière advient à soi comme un $A l s$ de ce Dass, et ce, à partir d'un point de soi qui reste hors de toute lumière. Ou si le $A l s$ est l'origine de la lumière, il l'est hors de toute

11. Ibid., p. 245 . 
lumière ${ }^{12}$; la lumière se pose, mais elle ne se pose que comme se présupposant; et ce se-présupposer est son Dass, un point qui dans la lumière reste toujours hors d'elle. De cette relation entre le $A l s$ et son Dass découle le moị réel comme quelque chose que l'on ne peut plus rechercher, comme quelque chose d'absolument inscrutable qui n'entre dans aucune lumière ${ }^{13}$. Et le moi est le retour de ce Dass à lui-même ${ }^{14}$. Et si l'existence absolue est l'existence de Dieu, le moi pur (ce pur se-poser en se présupposant) est le point de contact immédiat entre Dieu et son exister. Dieu même est immédiatement dans le moi, et il est le moi ${ }^{15}$.

Et si le moi en lequel nous nous convertissions avec la foi ne voyait pas sa propre nécessité interne et n'avait aucune nécessité, le moi pur que nous devenons maintenant dans ce venir du Dass à son $A l s$ - quand le Dass s"intellige, quand ce retour de l'exister divin à soi-même se produit - se possède évidemment lui-même en sa nécessité. Le Dass est nécessairement ce qu'il y a de premier, et il est nécessairement parce qu'il n'y a pas d'aspect qui ne le suppose une fois que le Dass est (mais comment pourrait-il ne pas être?). Le Dass est l'exister de l'absolu, l'« il y a » de l'absolu. Ce Dass est à proprement parler la Tat originaire, la Tathandlung dont il a été question dans la Doctrine de la Science de 1794-1795. Ce Dass n'est rien d'objectif ni d'objectivable. Il est l'objectivité même, absolue et pure, la projection pure ellemême, c'est-à-dire la projection à partir du néant. Il est, en somme, le savoir absolu qui, dans son devenir, précède comme factum tout savoir ${ }^{16}$.

Mais est-ce que, en parlant de cette façon, nous n'objectivons pas le Dass? Et est-ce que, par là-même, dans la mesure où il ne consiste pas en un exister absolu, il ne devient pas relatif à nous? La réponse de Fichte est négative. L'objectivation n'objective pas ce Dass, mais elle est précisément le $\mathrm{Als}$ de ce Dass dont nous dépendons. Ce Dass est le Dass du Als qui est seulement en tant que Als. L'exister absolu est cette objectivation même, comme factum fiens, que nous pouvons seulement appréhender sous la forme du Als ${ }^{17}$.

Dans la conférence 17, Fichte livre, de façon un peu subite, cette considération sur l'objectivité : "Moyennant son exister, l'absolu change son essence interne absolument, de manière irrécupérable, sans aucune possibilité de retour à soi-même dans l'exister, et moins encore dans l'existence, et

12. Ibid., p. 248.

13. Ibid., p. 249.

14. Ibid.

15. Ibid., p. 250.

16. Ibid., p. 253.

17. Ibid., p. 255. 
ce changement est immédiatement l'objectivité ${ }^{18}$. "Mais, d'un autre côté, Fichte ajoute: "c'est l'absolu qui existe, et aucun autre à sa place, car il ne pourrait devenir un autre hors de lui ${ }^{19}$. " Nous avons, par conséquent, identité dans la non-identité et non-identité dans l'identité. "C'est l'objectivité comprise en son essence. "Et qu'est-ce que c'est - sinon le concept? L'objectivité est en son essence concept, en opposition à l'identité fermée de l'être en soi ${ }^{20}$. Et si cette essentielle identité (Dieselbigkeit) dans une essentielle non-identité, dans son unité qualitative, est le concept, alors l'absolu existe seulement comme concept puisque l'exister absolu n'est que l'exister de l'absolu.

Dans les conférences 18 et 19, Fichte fait appel à la distinction traditionnelle entre le concept objectif ou l'essence de la chose (la forme existentielle interne de la chose, ses loi et nécessité internes) et le concept subjectif, c'està-dire le concept en tant que concept, le savoir. L'exister de Dieu est le concept originaire (Urbegriff), il est la pré-construction de ce dont le savoir est la post-construction. Et Fichte a confiance dans le fait que les auditeurs ont désormais appris à bien distinguer entre le noumenon (l'absolu, Dieu), le monde pris absolument (le concept originaire, l'exister de Dieu) et le savoir ou l'existence (le $A l s$ ), et surtout à ne pas mettre le noumenon dans le monde pris absolument, c'est-à-dire dans la sphère de la pré-construction, et à ne pas confondre la pré-construction avec la post-construction, ce qui donnerait lieu à un subjectivisme ridicule avec lequel Fichte n'a absolument rien à voir ${ }^{21}$.

L'être est l'identique face à la différence de l'être avec soi-même dans le concept. Mais, quelle est cette différence ou en quoi consiste-t-elle lorsqu'il s'agit de l'absolu et du concept originaire qui est l'exister de l'absolu? Fichte n'a pas beaucoup à dire sur ce sujet, et sa réponse est paradoxale: "Posé l'exister, il faut que ['absolu existe] - il reste par conséquent la liberté de l'exister et un maillon préalable à la nécessité. [/] Visiblement le concept de l'essence laisse certaines choses sans les appréhender, à savoir: le libre, le contingent qui ne peut pas être posé en vertu de la loi. - Par conséquent, le concept laisse dans l'être une non-identité avec soi ${ }^{22}$. "Dans l'absolu, cette liberté est l'exister même comme factum. Mais s'il en est ainsi, nous nous voyons alors renvoyés une fois encore à la problématique de la treizième conférence sur la foi, et à travers elle, à la problématique de la septième
18. Ibid., p. 257.
19. Ibid.
20. Ibid., p. 258.
21. Ibid., p. 268.
22. Ibid., p. 261. 
conférence. Fichte a raison alors, dans la seizième conférence, de se plaindre du fait que l'exposé commence à prendre l'aspect d'un constant mouvement de retour sur lui-même, comme cela lui était déjà arrivé à plusieurs reprises.

Mais il ne s'agit pas seulement de cela. Fichte n'a pas été non plus capable de répondre à une autre question qu'il s'était déjà posée plusieurs fois au cours de l'exposé (et que le lecteur s'est, sans doute, déjà posée lui-même) et qui, dans la conférence 18 précisément, devient inévitable: Qu'est-ce donc que l'absolu? Si Dieu existe uniquement en tant que concept et si le concept est l'essence, le quoi de la chose, alors il faut se demander: comme quoi Dieu existe-t-il, quel est le concept de Dieu ou le concept originaire de Dieu? Car il ne suffit pas de répondre qu'il existe comme fondement parce qu'il faut se demander également quelle est l'essence de ce fondement, das Wesen des Grundes, une fois qu'il n'existe que comme concept. Fichte se limite à répondre: "Puisque cet exister est immédiat, on ne peut l'appréhender que de façon immédiate, en devenant ce concept et en l'étant; de telle sorte qu'il n'y a plus rien à dire. Et la doctrine de la science se gardera bien de dire autre chose $^{23}$."

Quatrième tentative: l'absolu sans quoi et l'existence comme rien

Malgré ce que Fichte affirme dans la conférence 18, les conférences 21 à 25 ne développent pas de thème nouveau, mais constituent une quatrième tentative de résoudre l'aporie à laquelle la troisième tentative avait donné lieu. La reconstruction de l'argumentation de Fichte est ici particulièrement difficile parce que, d'une part, Fichte l'énonce sans aucun plan déterminé et, d'autre part, parce qu'il semble aussi changer d'opinion et même de langage à mesure que les difficultés surgissent.

Nous pouvons partir d'une formulation de la conférence 21. Nous avons distingué deux moments dans l'existence, le Dass et le $A l s$, ou une double forme de l'exister: absolument et en tant qu'absolu. Mais comme le Dass est le Dass du Als, l'absolu ne peut pas exister adverbialiter sans exister en tant $q u$ 'absolu. Or, si nous envisageons son exister adverbialiter, l'absolu existe absolument mais pas en tant qu'absolu, c'est-à-dire sans se laisser interpréter en tant qu'absolu. Et comme il ne peut pas exister s'il n'existe pas en tant qu'absolu (puisque l'existence est $A l s$ ) et que l'existence est relation, l'absolu, en tant qu'absolu, n'est pas absolu, mais seulement relatif. Et, par conséquent, il n'existe pas absolument. «L'absolu se déchire lui-même dans 
son exister: en exister absolu - et alors il n'existe pas en tant qu'absolu -, et en exister en tant qu'absolu - et alors il n'existe pas absolument ", mais seulement relativement ${ }^{24}$, et cela veut dire qu'absolument il n'existe pas, ou ce qui revient au même: que l'existence absolue (le savoir) ne pourrait se comprendre en tant qu'existence de l'absolu. Devant une telle autonomie de l'existence face à l'absolu dont elle est l'existence, comment pourrait-on encore comprendre l'existence absolue en tant qu'existence de l'absolu, ou qu'est-ce que l'existence absolute sumpta? La réponse a déjà été donnée dans la septième conférence: la lumière, en comprenant son en-soi comme l'existence d'un étranger, se comprend elle-même en tant que non-être, en tant que rien face à l'absolu dont elle est l'existence; c'est-à-dire, l'existence n'étant rien, il s'ensuit qu'elle n'est aucun milieu de déchirement. Autonomie de l'existence, se comprendre en tant que rien, et se comprendre sont les trois motifs principaux de cette quatrième tentative.

AUTONOMIE DE L'EXISTENCE. - Fichte fait appel à la structure A/A x A d'après laquelle l'existence ne place devant elle son propre être $(x A)$ qu'en étant en relation avec elle-même (A/A). Et il interprète $\mathrm{A} / \mathrm{A}$ en termes d'intuition (Anschauung) et d'entendement (Verstand). L'entendement, en se voyant, se comprend (A/A) et il comprend son être (x A) comme rien. Si l'existence est l'existence de l'absolu, c'est-à-dire si l'existence est l'absolu en tant qu'absolu, "cet $A l s$ est en vertu de son être formel une relation, et moyennant cette relation, est posé un deuxième membre par le biais duquel n'est pas compris l'absolu, mais l'intuition (A) de l'absolu. La forme de l'exister divin est ici intuition, ou raison et entendement en unité; mais elle est entendement seulement médiatement, et en relation avec la raison, de sorte que c'est seulement l'intuition qui se comprend elle-même comme intuition de l'absolu. Et Dieu n'entrerait pas en contact avec l'entendement de façon immédiate, mais seulement au moyen de la raison ${ }^{25}$. "

Mais la question est de savoir $\mathrm{si}$, dans tout ce que nous venons de dire et qui laisserait l'absolu hors du $A l s$, il y a un autre absolu que le caractère absolu lui-même de la lumière. Comme il faut répondre qu'il y n'en a pas, il semble que la notion d'absolu devient une fois encore, comme cela s'est déjà produit dans les conférences 7 et 8 , une confusion de la lumière avec ellemême. Fichte de protester: "L'absolu dans la forme, donc mêlé avec elle, sans possibilité d'écart. Que la possibilité de la conscience se fonde dans la possibilité de cet écart et que la possibilité du monde repose dans cette possibilité, c'est là quelque chose que nous savons déjà depuis un certain 
temps ${ }^{26}$. "Il semble que l'autonomie de l'existence nous ramène une fois encore, non seulement à la septième conférence, mais même au tout début: à la question de savoir comment comprendre le chiasme entre être et existence.

Se COMPRENDRE EN TANT QUE RIEN. - Quoi qu'il en soit, de quelle autre façon la lumière pourrait-elle comprendre son propre être face à l'absolu si ce n'est comme rien? Qu'est-ce que l'existence de l'absolu sinon l'absolu en tant que tel? " Je vous invite à reconnaître ce que vous pouvez reconnaître par l'intuition intellectuelle seule, à savoir: que l'absolu dans la forme du $A l s$ est absolument vide. De fait, que signifie ce $A l s$, si ce n'est que l'absolu est l'absolu, et rien de plus? Dans la forme du $A l s$, l'absolu est dans un simple représentant, sans aucun contenu; son contenu n'est rien, simplement un absolu formel. - En tant qu'absolu: et en général avec tout $A l s$, lui ajoutet-on quelque chose? Cette forme est, par conséquent, absolument vide et ne donne rien. Je l'ai appelée une simple répétition numérique ${ }^{27}$."

Or, «cette vacuité est le précipité de l'intuition, un dépôt d'elle-même ». Car, " comment l'intuition se comprend-elle? Manifestement comme un rien absolu, puisque dans son produit, elle reconnaît ne rien produire. Pour la même raison, elle sépare d'elle l'absolu comme le réel et elle le projette comme n'étant ce rien, en ne le tolérant point dans le rien ni confondu avec ce rien. [...] Et son comprendre est identique à la manière dont il se comprend en se comprenant lui-même - à savoir rien précisément; et en séparant le quoi, et en lui laissant l'empreinte de son rien " ${ }^{28}$. "Une autre question - ajoute Fichte - est de savoir comment ce quoi entre dans le rien pour être séparé du rien moyennant le rien ${ }^{29}$."

SE COMPRENDRE. - L'existence n'est qu'en tant qu'existence, c'est-àdire, "de manière absolue, la lumière est seulement en se comprenant, et elle se pose seulement par cette auto-compréhension [...]. Par conséquent, à l'être absolu de la lumière, il ne faut pas lui en faire précéder un autre [...], mais c'est seulement moyennant ce même comprendre que la lumière, de manière originelle et créatrice, pose comme présupposé un être indépendant du Verstehen même ${ }^{30}$ ». Et «si, en ce se-comprendre, la lumière se comprend comme rien, la lumière présuppose son être interne comme rien [...]. Or, la compréhension comme telle n'est possible que par une opposition, donc, la compréhension de ce rien n'est possible que par opposition à la notion de

26. Ibid., p. 278.

27. Ibid., p. 280.

28. Ibid.

29. Ibid., p. 280.

30. Ibid., p. 282 sq. 
quelque chose, à la notion de réalité. Par conséquent, pour l'auto-intuition de son rien, il est nécessaire que la lumière ait devant elle quelque chose, ou la réalité ${ }^{31}$. " Et quelle autre réalité la lumière a-t-elle ici devant elle, si ce n'est sa propre réalité en ce qu'elle comprend cette réalité comme un rien? La comprendre comme un néant: mais face à quoi? Face à rien.

Si l'auto-compréhension de la lumière est ce dont tout doit à présent surgir ${ }^{32}$, il faut alors reconnaître ${ }^{33}$, dit Fichte, que ce qui nous est arrivé au point auquel nous nous trouvons est que, si la compréhension ou la lumière que nous sommes devenus ne se comprend pas, en elle-même, comme un rien (et comment pourrait-elle se comprendre comme rien, si elle est précisément compréhension, et par conséquent quelque chose?), alors l'absolu comme tel n'est point un absolu, mais quelque chose de relatif à la lumière. Et si la lumière, en elle-même, comprend son être comme rien (et le Dass et le $A l s$ ne semblent pas être autre chose que ce rien), alors on ne voit point le quoi face auquel l'existence pourrait se comprendre comme n'étant rien, sauf le quoi de l'existence elle-même. Mais comment l'existence pourraitelle se comprendre comme rien vis-à-vis de son propre quoi?

\section{Cinquième tentative: l'autonomie de l'existence et l'absolu comme loi}

Fichte introduit ce concept difficile d'une manière quelque peu étrange. "L'absolu est le fondement du Dass; pure loi de ce que cette forme existe ${ }^{34}$. " Si la loi est une loi du pur Dass, cela assure la complète autonomie de la forme. Étrangement, l'absolu serait le fondement qui ferait que son existence soit facticité radicale, grundlose Wirklichkeit, c'est-à-dire nonabsolue, à moins que la loi signifie que ce Dass se produit nécessairement; étrangement, l'essence de Dieu consisterait purement et simplement en la nécessité de ce Dass.

Nous avons déjà vu dans la septième conférence que, dans la lumière, son Dass est nécessaire, pour peu que la lumière soit. Fichte affirme d'abord cette nécessité sous la forme d'une version nihiliste de l'argument ontologique: de l'essence de l'absolu découle nécessairement son existence. - Dans la conférence 24, dans l'un des passages les plus célèbres de cet exposé, Fichte dit: "Il réside dans l'essence interne purement spirituelle de Dieu que, face à elle, le rien se comprend comme rien, et que précisément, en se

\footnotetext{
31. Ibid, p. 283.

32. Ibid., p. 284.

33. Ibid., p. 285.

34. Ibid., p. 287.
} 
comprenant comme rien et simplement à travers cette compréhension, le rien se configure et se crée comme quelque chose d'apparent; et de cette manière, il peut intuitionner l'absolu moyennant une intuition qui, cependant, reste éternellement vide, dont toute la réalité est seulement celle qui a été mentionnée plus haut: à savoir que c'est dans l'essence divine que réside intérieurement la nécessité de cette intuition. Ce qui existe absolument par soi-même et en vue de soi-même, et qui est absolument nécessaire, de la même manière que l'absolu lui-même, c'est l'intuition de Dieu : la création absolue de soi du néant est seulement la condition extérieure de possibilité, c'est-à-dire la forme originaire de cette intuition. Le monde se crée absolument lui-même, et c'est précisément dans cette création, dans cette genèse à partir du rien, que réside l'inéliminable trace de son rien, puisque rien n'advient du rien: par opposition à l'être de l'absolu, qui ne devient pas, mais repose absolument en lui-même. Rien n'advient du rien; le rien reste éternellement rien: ces propositions ont ici - et ne peuvent avoir - une validité stricte qu'à ces conditions ${ }^{35}$. "

Critique de l'argument ontologique. - Mais ensuite, dans la conférence 25 , où il fait allusion à l'argument ontologique, Fichte annule cette notion de nécessité. L'existence comme telle ne se comprend pas comme conséquence - on l'a dit dans la septième conférence -, elle n'est rien d'autre, en réalité, que l'identité de l'absolu lui-même, une identité sans différence, qui devient visible précisément par la voie de l'auto-négation de la conséquence. "On dit qu'en Dieu l'existence découle de son être interne. Elle en découle: comme s'ils fussent deux, alors qu'ils sont seulement un ${ }^{36}$. "Alors pourquoi ce elle-en-découle, pourquoi cette conséquence? Fichte répond: manifestement, parce que l'absolu est pris dans la forme de la conséquence; on le confond avec elle et on le rend identique à elle, et il reste en elle déchiré en deux; mais cela n'est pas ainsi, parce que, comme nous l'avons répété, la conséquence s'anéantit comme conséquence. Dans son caractère absolu, la forme se pose comme s'anéantissant, et elle s'anéantit en son auto-position absolue. "La relation fondement-conséquence est portée absolument par l'existence même, par la conséquence même, et, par suite, d'après elle-même; dans l'absolu même, il ne peut y avoir ni conséquence, ni nécessité, ni rien de tout cela. [...] Mais ici, la conséquence s'anéantit comme conséquence (en ce qui concerne sa validité), et elle laisse seulement sa facticité ${ }^{37}$. " L'absolu est pure identité: "Dieu est-il créateur du monde? Pas du tout, parce qu'il n'y a aucun monde et qu'il ne peut point 
y en avoir; car seul l'absolu est; et l'absolu ne peut pas réellement, ni véritablement, sortir de soi-même ${ }^{38}$. Par conséquent, le est qui dément cette identité ne peut être autre chose que le néant.

D'une part, donc, le savoir, en lui-même, en se heurtant à son propre ensoi, cesse d'être savoir et doit regarder son propre être comme existence d'autrui, comme existence non seulement d'une énigme, mais d'un étranger absolu dont il doit nier tout quoi. D'autre part, le savoir doit comprendre son propre être comme un rien face à un absolu qui semble ne pas être autre chose qu'un simple concept qui naît et se nourrit de cette opposition et qui, par suite, est aussi relatif à la lumière. Et, de plus, se-comprendre est justement se comprendre, c'est-à-dire, se comprendre est quelque chose, qui a comme centre le se du se-comprendre. Et ce se précède l'aspect du se comprendre comme quelque chose et l'aspect du se comprendre comme rien.

Fichte consacre alors les conférences 26, 27 et 28 à l'analyse de ce $s$. Malgré toutes les subtilités et au terme de nombreux va-et-vient, la conclusion ne diffère pas de ce que Fichte avait déjà dit dans la septième conférence en parlant de la lumière comme relation: la lumière est un voir auquel s'ajoute un autre voir, une relation dans laquelle les relata et le fondement de la relation sont la relation elle-même, sont une seule et même chose; un voir qui jaillit comme étant lié à son propre être, lequel n'est rien d'autre qu'un voir; comme étant lié à sa propre forme (Gebundenheic), comme n'étant lié qu'en jaillissant comme étant lié à sa propre forme, en se posant comme jaillissant comme étant lié à sa propre forme (Ungebundenheit, Freiheit). La lumière est auto-sentiment; en se posant comme jaillissant comme étant lié à sa propre forme, elle est un se-comprendre.

Mais que veut dire ce se comprendre comme rien? - se demande finalement Fichte dans la conférence 28. Il ne sait pas très bien que répondre à cette question. Il use d'un argument qui n'est pas acceptable parce qu'au point où nous nous trouvons, on ne peut pas introduire comme prémisse la proposition selon laquelle seul l'absolu existe et que c'est seulement en comprenant son propre être comme rien que l'existence peut se comprendre comme existence de l'absolu. Or c'est précisément ce que, dès la septième conférence, Fichte veut démontrer - mais sans pourtant y parvenir. "La connaissance rationnelle de l'absolu est qu'absolument en dehors de lui, il n'y a rien. Par conséquent, il faut anéantir cela même dans la connaissance rationnelle, déduite précédemment, qui se montre comme un produit de l'entendement : à savoir le quoi par opposition au rien de l'entendement qui se perçoit soi-même, un produit de l'opposition et, par conséquent, de la

38. Ibid., p. 288. 
dualité. [...] L'intuition est l'anéantissement absolu de la forme de l'entendement à même la raison par la raison pure elle-même [...], et derechef l'anéantissement de celle-ci est l'intuition. [...] Et si maintenant l'intuition s'anéantit elle-même, alors il ne reste qu'un être hors duquel il n'y a absolument rien, et qui est justement égal à l'absolu ${ }^{39}$. " Comment l'intuition s'anéantit-elle? En ce que, par elle-même, elle ne signifie rien, mais que seul l'absolu signifie quelque chose en elle - qui est le vrai au sens du réel. "Le factum de la vérité, qui n'a aucune vérité, est l'intuition. Et cette intuition, afin que le factum soit la vérité, doit se comprendre comme un rien. Et c'est précisément la raison pour laquelle il doit se comprendre comme un simple factum ${ }^{40}$ ", comme grundlose Wirklichkeit, pur apparaître sans fondement. Et c'est cela que ce rien signifie pour Fichte; mais sans un quoi opposé à ce rien, un quoi qui entre dans le savoir, on ne voit pas comment le savoir (ce factum) peut contenir un inconditionné, c'est-à-dire comment un savoir pourrait être véritablement, et non pas simplement être un apparaître sans fondement, une pure apparence.

Fin de la cinquième tentative: liberté et loi

Ainsi en arrivons-nous à la conférence 29 qui, compte tenu du fait que l'exposé se termine avec elle, prend l'allure d'une conclusion et d'une récapitulation. Dans l'exposé, Fichte a cherché à montrer que le savoir, que nous devons comprendre comme existence absolue, ne peut être autre chose que l'existence de l'absolu. Mais il n'a pas réussi à le démontrer. Il tente alors une dernière fois - en commençant à nouveaux frais - d'esquisser une issue possible.

S'il s'agit d'expliquer ce qu'est le savoir, c'est-à-dire s'il s'agit d'arriver au centre de la division entre l'être et l'existence, nous ne devons pas commencer en présupposant quelque chose qui puisse seulement être le produit de cette division. Prenons les deux idées suivantes. La première: si nous posons une image de l'absolu, cette image devra être quelque chose de réellement autonome, de libre, de soi, par soi, en soi. La seconde: cette image ne sera pas à son tour une image, mais l'absolu lui-même, si elle ne se fait pas image de soi comme image.

Au cours de l'exposé, ces deux idées - qui résument très bien tout son contenu - n'ont pas évité le soupçon de n'être qu'un pur produit de la pensée, au sens d'une confusion de la pensée avec elle-même; et, par conséquent,

39. Ibid., p. 306.

40. Toid. 
elles ne peuvent pas devenir le principe de la doctrine de la science, si nous ne voulons pas éviter de nous mouvoir en cercle.

Et Fichte de se demander: comment sortir de ce cercle? Il répond: en présupposant une loi absolue de penser tel que nous avons pensé. « Cette loi est l'unique fondement du savoir par lequel est posé tout ce qui est posé dans le savoir; elle est l'unique manière, et toute la manière, selon laquelle l'absolu entre dans le savoir ${ }^{41}$. " Mais pourquoi? Pour quelle raison ne pourraitelle pas être également produite par la pensée?

Cette loi est donc la loi de penser tel que nous avons pensé. Et comment avons-nous pensé? Nous avons pensé en concluant à chaque fois sur l'affirmation de l'autonomie de l'existence, et en réduisant la raison à un pur factum, à la grundlose Wirklichkeit, en effaçant successivement tout quoi audelà de l'existence dont l'existence pourrait être l'existence. Et on peut affirmer que cela n'est pas seulement le résultat de l'exposé, mais de la philosophie transcendantale en général et de celle de Fichte en particulier. Ce résultat, comme Fichte l'a expliqué dans la conférence 27 , a continuellement été un se-trouver de l'existence, c'est-à-dire de la liberté, comme jaillissant de rien, liée à son propre être, sans fondement; car la liberté doit en effet cette absoluité à son caractère de Nicht-Folge, à sa Grundlosigkeit, à sa facticité.

Mais cela veut dire que la raison moderne occupe le lieu d'un absolu, tout-à-fait effacé, que, cependant, elle ne saurait être. Si elle existe, ou une fois qu'elle est, elle est certainement un absolu, mais elle ne peut pas suffire à cette condition. En étant mise à la place de cet absolu (un absolu qui manque de tout quoi), qu'elle ne peut être, en étant en son lieu, la liberté est l'unique représentation, image, Bild de cet Autre. La raison ne peut se comprendre, dans son autoposition absolue, que comme la représentation ou l'image de l'absolu qu'elle n'est pas; elle est seulement en son lieu, sans qu'il y ait de cet absolu une autre image qui ne soit elle; " et ce que nous nommons une image, n'est-ce pas ce qui n'est pas ${ }^{42}$ ? "

C'est justement parce que, en dépit du fait de n'être point l'absolu effacé - lieu en lequel elle est placée -, la raison ne peut qu'affirmer sa propre inconditionnalité, qu'elle ne peut pas s'en défaire. Par conséquent, si la raison doit elle-même se prendre au sérieux dans son caractère ultime et dans son caractère de Nicht-Folge, cet inconditionné même n'existe que moyennant le factum de l'auto-position absolue de la raison. Au fond, le factum de . la vérité n'a aucune vérité face à la vérité. Mais comme la facticité de l'exis-

41. Ibid., p. 308.

42. Platon, Le sophiste, $240 \mathrm{~b}$. 
tence est la condition de l'être-là de la vérité, ce qu'il peut y avoir d'inconditionné dans le moi, dans un sens autant théorétique que pratique, «est seulement cela: que la liberté soit, afin qu'elle se comprenne soi-même, comme image de Dieu ${ }^{43}$ ", comme l'inscrutable existence de l'inconditionné.

Résumé: Cet article propose une reconstruction de la ligne d'argumentation de l'exposé de 1805 de la Doctrine de la Science de Fỉchte. Son objet fondamental consiste à répondre d̀ la question: qu'est-ce que le savoir? ce qui implique de répondre à cette autre question: qu'est-ce que le " est "? (contenue dans la question qu'est-ce que le savoir?) Fichte répond: ce savoir est l'existence absolue, ce qui est équivalent à l'existence de l'absolu (Dieu)-et l'auteur le montre à travers cinq tentatives de démonstration de cette thèse. Cela aboutit à la compréhension du caractère inconditionné de la raison (= factum de l'auto-position absolue de cette dernière), résultat de la philosophie transcendantale en général.

Mots-clés: Existence. Savoir. Absolu. Raison.

Abstract: This paper proposes a reconstruction of the argumentative line of the exposition of Fichte's Science of Knowledge of 1805. Its fundamental object consists in answering the question: what is knowledge? what implies to answer another question: what 'is' is? (included in the question what is knowledge?) Fichte answers that knowledge is absolute existence, what is equivalent to the existence of absolute (God) - and the author demonstrates his thesis through five attempts. It ends up at the comprehension of the unconditioned character of reason ( = factum of the absolute self-positing of the reason), result of the transcendental philosophy in general.

Key words: Existence. Knowledge. Absolute. Reason. 